\title{
JENSEN SHANNON DIVERGENCE AS A MEASURE OF THE DEGREE OF ENTANGLEMENT
}

\author{
A.P. Majtey, A. Borras, M. Casas \\ Departament de Fisica and IFISC-CSIC, \\ Universitat de les Illes Balears \\ Palma de Mallorca, 07122, Spain \\ ana.majtey@uib.es, toni.borras@uib.es,montse.casas@uib.es \\ P.W. Lamberti \\ Facultad de Matemática, Astronomía y Física, \\ Universidad Nacional de Córdoba and CONICET \\ Córdoba, 5000, Argentina \\ lamberti@famaf.unc.edu.ar \\ A. Plastino \\ IFLP-Facultad de Ciencias Exactas, \\ Universidad Nacional de La Plata and CONICET \\ La Plata, 1900, Argentina \\ plastino@fisica.unlp.edu.ar
}

\begin{abstract}
The notion of distance in Hilbert space is relevant in many scenarios. In particular, "distances" between quantum states play a central role in quantum information theory. An appropriate measure of distance is the quantum Jensen Shannon divergence (QJSD) between quantum states. Here we study this distance as a geometrical measure of entanglement and apply it to different families of states.
\end{abstract}

\section{INTRODUCTION}

Discerning possible candidates for measuring distances between quantum states is a subject of perennial interest. Many of these measures were first defined as distances between probability distributions and subsequently employed as distance-measures in Hilbert space. Let $\mathcal{H}$ be the Hilbert space associated with a quantum system and let $\mathcal{S}$ be the set of all states, i.e. the set of self-adjoint, (semi)positive and trace-one operators. A frequently employed notion of distance between quantum states is the relative entropy, which is a natural extension to the realm of quantum mechanics of the Kullback-Leibler divergence. This quantity, however, is not useful for ascertaining the degree of purification of an arbitrary state with respect to a pure reference state. The relative entropy of an operator $\rho$, with respect to an operator $\sigma$, both belonging to $\mathcal{S}$, is given by

$$
S(\rho \| \sigma)=\operatorname{tr}[\rho(\log \rho-\log \sigma)],
$$

where $\log$ stands for logarithm in base two. $S(\rho \| \sigma)$ is nonnegative and vanishes if and only if $\rho=\sigma$, being nonsymmetric and unbounded. A particular and important requirement indicates that the relative entropy is well defined only when the support of $\sigma$ is equal to or larger than that of $\rho$. Otherwise, it is defined to be $+\infty$ [1] (the support of an operator is the subspace spanned by its eigenvectors with non-zero eigenvalues). To overcome this restriction we have introduced a distance between elements of $\mathcal{S}$ that shares with the relative entropy several of their main properties but that is always well defined and bounded [2]. This distance is the quantum Jensen-Shannon divergence (QJSD), which is a quantum mechanical extension of the Jensen-Shannon divergence (JSD) introduced by Rao [3] and Lin 4] as a distance between probability distribution (for a detailed analysis of the properties of the JSD, see reference [5]). Here we wish to investigate the ability of the QJSD to serve as a measure of the degree of entanglement.

The structure of the paper is as follows. In the next Section we review the basic properties of the QJSD. In Section 3 we study its properties as an entanglement measure and we apply it to quantify the degree of entanglement of different families of two-qubit mixed states. Finally, some conclusions are drawn in Section 4.

\section{THE QUANTUM JENSEN-SHANNON DIVERGENCE}

We define the QJSD in the fashion [2]

$$
J S(\rho \| \sigma)=\frac{1}{2}\left[S\left(\rho \| \frac{\rho+\sigma}{2}\right)+S\left(\sigma \| \frac{\rho+\sigma}{2}\right)\right],
$$


which can be also recast in terms of the von Neumann entropy $H_{N}(\rho)=-\operatorname{tr}(\rho \log \rho)$ as follows

$$
J S(\rho \| \sigma)=H_{N}\left(\frac{\rho+\sigma}{2}\right)-\frac{1}{2} H_{N}(\rho)-\frac{1}{2} H_{N}(\sigma) .
$$

This quantity has a lot to speak for, being positive, null iff $\rho=\sigma$, symmetric, bounded, and always well defined. In fact, the restriction imposed on the supports of $\rho$ and $\sigma$ for the relative entropy (2) is lifted for the QJSD, that possesses all the adequate properties of a proper distance between states in a Hilbert space. As stated above, in this work we attempt to study the QJSD as an entanglement measure which justifies listing the main QJSD properties. Most of these properties are discussed and proved in Ref. [2]. The list reads

(i) $J S(\rho \| \sigma) \geq 0$ with the equality iff $\rho=\sigma$

(ii) Unitary operations left JS invariant, i.e., $J S\left(U \rho U^{\dagger} \| U \sigma U^{\dagger}\right)=J S(\rho \| \sigma)$.

(iii) $J S\left(\operatorname{tr}_{p} \rho \| \operatorname{tr}_{p} \sigma\right) \leq J S(\rho \| \sigma)$ where $\operatorname{tr}_{p}$ is the partial trace.

(iv) JS is jointly convex $J S\left(\sum_{i} \alpha_{i} \rho^{(i)} \| \sum_{i} \alpha_{i} \sigma^{(i)}\right) \leq \sum_{i} \alpha_{i} J S\left(\rho^{(i)} \| \sigma^{(i)}\right)$, where the $\alpha_{i}$ are positive real numbers such that $\sum_{i} \alpha_{i}=1$.

(v) $J S(\Phi \rho \| \Phi \sigma) \leq J S(\rho \| \sigma)$ for all positive mappings $\Phi$.

(vi) For any set of orthogonal projectors $P_{i}$, such that $P_{i} P_{j}=\delta_{i j} P_{i}, J S\left(\sum P_{i} \rho P_{i} \| \sum P_{i} \sigma P_{i}\right)=\sum J S\left(P_{i} \rho P_{i} \| P_{i} \sigma P_{i}\right)$

(vii) $J S\left(\rho \otimes P_{\alpha} \| \sigma \otimes P_{\alpha}\right)=J S(\rho \| \sigma)$ where $P_{\alpha}$ is any projector.

The two last properties, which have not been discussed before, are verified by the relative entropy [1, 6], and inherited by the QJSD.

In a recent work [7] the metric character of the QJSD has been discussed. There it was formally proved for pure states, and checked numerically for mixed ones by performing Monte Carlo simulations. We can thus assert that the square root of the QJSD verifies the triangle inequality, as it does the square root of the JSD.

\section{THE ENTANGLEMENT MEASURE}

Entanglement constitutes a physical resource that lies at the heart of quantum information processes [8, 9, 10]. Quantum teleportation, superdense coding, and quantum computation, are some of the most representative examples. Let us recall that a state of a composite quantum system is called entangled if it can not be expressed as a convex sum of factorizable pure states. Otherwise, the state is called separable.

Nowadays a variety of measures are used to quantify the degree of entanglement. These include the entanglement of distillation, the relative entropy of entanglement, etc. The canonical measure of entanglement in a bipartite pure systems is the so-called entanglement of formation, which is a strictly monotonic function of the squared concurrence. For simplicity the entanglement of formation is frequently used as the measure of entanglement. However, for mixed state there exist several available measures.

Before starting with the entanglement-characterization using the QJSD we study the structure of Hilbert space (HS) according to this distance measure. To do that we evaluate $d_{J S}=\sqrt{J S}$ between a given random generated state and the maximally mixed (MM) state $\rho_{M M}=I / N$, with $N$ the HS-dimension. In Fig. 1 we plot the probability distribution for finding an arbitrary state $\rho$ at a given distance from the $\rho_{M M}$. We find, as expected, that the mean value of such distance increases for higher Hilbert space-dimensions.

Let us now enumerate the properties that any adequate measure of entanglement should satisfy [11]:

(i) Discrimination: $\mathcal{E}(\rho)=0$ iff $\rho$ is separable.

(ii) Monotonicity: the measure does not increase under local general measurements and classical communication, for every completely positive map $\Phi, \mathcal{E}(\Phi \rho) \leq \mathcal{E}(\rho)$

(iii) Convexity: $\mathcal{E}(x \rho+(1-x) \sigma) \leq x \mathcal{E}(\rho)+(1-x) \mathcal{E}(\sigma)$, with $x \in[0,1]$.

As it was already stated, the main purpose of the present communication is to investigate the QJSD as a geometrical measure of entanglement. Following Vedral et al., [12] we define an entanglement measure $\mathcal{E}(\rho)$ as the minimum QJSD from the state $\rho$ to the set $\mathcal{D}$ of the disentangled states.

$$
\mathcal{E}_{J S}(\rho)=\min _{\sigma \in \mathcal{D}} J S(\rho, \sigma) .
$$


The QJSD properties enunciated in the preceding section ensure that (4) fulfills the conditions for an adequate entanglement measure. For convenience's sake, we normalize the entanglement measure by a trivial re-scaling in order to adequately compare different quantities of interest, i.e., $\mathcal{E}(\rho)=\mathcal{E}(\rho) / \mathcal{E}\left(\left|\psi^{-}\right\rangle\left\langle\psi^{-}\right|\right)$.

It is notheworthy to stress that expression (4) has already been investigated for different distances, other than the JS-one. For example, Vedral and coworkers used to this effect the relative entropy $\left(\mathcal{E}_{R E}(\rho)\right)$ and the Bures metric $\left(\mathcal{E}_{B}(\rho)\right)[6,12]$. Also the Trace-distance and the Hilbert-Schmidt metric have received consideration for the purpose [13, 14]. We shall below use as a reference the quantity $\mathcal{E}_{B}(\rho)$. In order to investigate the behavior of the QJSD as

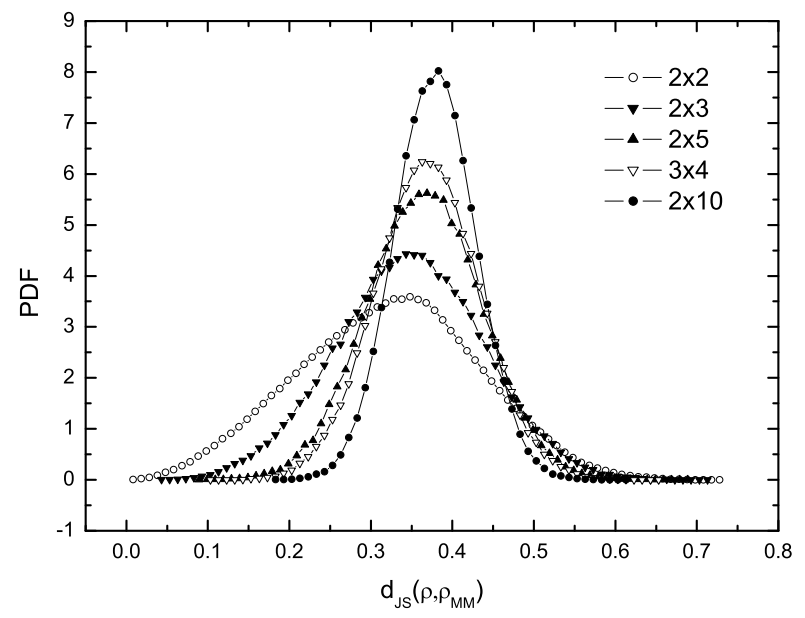

FIG. 1: Probability distribution for finding a quantum state at a given distance from de maximally mixed one (for different Hilbert space dimensions).

an entanglement measure we consider now three well-known families of two-qubits mixed states, namely,

- Werner ones [15],

- maximally entangled mixed ones [16] (MEM), and

- parametric down-conversion [17] (PDC) states.

All of these are diagonal in the Bell basis. We perform an optimization procedure so as to find the separable state that lies in the closest proximity to the state of interest $\rho$. Such task is performed by following a simulated annealing minimization procedure, starting from the maximally mixed state $\rho_{M M}$. Appropriately perturbing $\rho_{M M}$ we look for the minimal QJSD between $\rho$ and some state in $\mathcal{D}$.

In Fig.2 we depict, versus the linear entropy $S_{L}$ (the degree of impurity), i) the concurrence and the two normalized quantities: ii) our $\mathcal{E}_{J S}$ and iii) Vedral's $\mathcal{E}_{B}$ measure. This is done for the three families enumerated above.

Notice that the concurrence and $\mathcal{E}_{B}$ are greater than $\mathcal{E}_{J S}$, except for the "extreme" cases of maximally entangled or disentangled Bell states in two of the sub-figures. In the MEMs-instance this is not entirely so. Crossings are detected between $\mathcal{E}_{B}$ and the concurrence $\mathcal{C}$, although the $\mathcal{E}_{J S}$-curve lies always below the other two for all the three families, except for the extreme (trivial) situations. The behavior here described mimics that of the relative entropy vs. $S_{L}$. In that instance, the relative entropy-induced entanglement is strictly smaller than that of formation, as analytically demonstrated in [6].

Our crucial point is the fact that the measure induced by the QJSD is easier to compute than that induced by the Bures metric. This is so because of the square root in the definition of Bures' metric, which forces cumbersome matrix-diagonalization and basis' changes in order to compute $\mathcal{E}_{B}$. Instead, so as to calculate the QJSD one i) only needs to solve the eingenvalue-equation for three matrices (eigenvectors are not necessary) and ii) the optimization procedure is much more efficient than in the Bures situation.

\section{CONCLUSIONS}

We have here advanced a new entanglement-measure $\mathcal{E}_{J S}$, based on the Jensen-Shannon distance. For three important families of states this new quantity behaves in rather similar fashion as the established entanglement- 

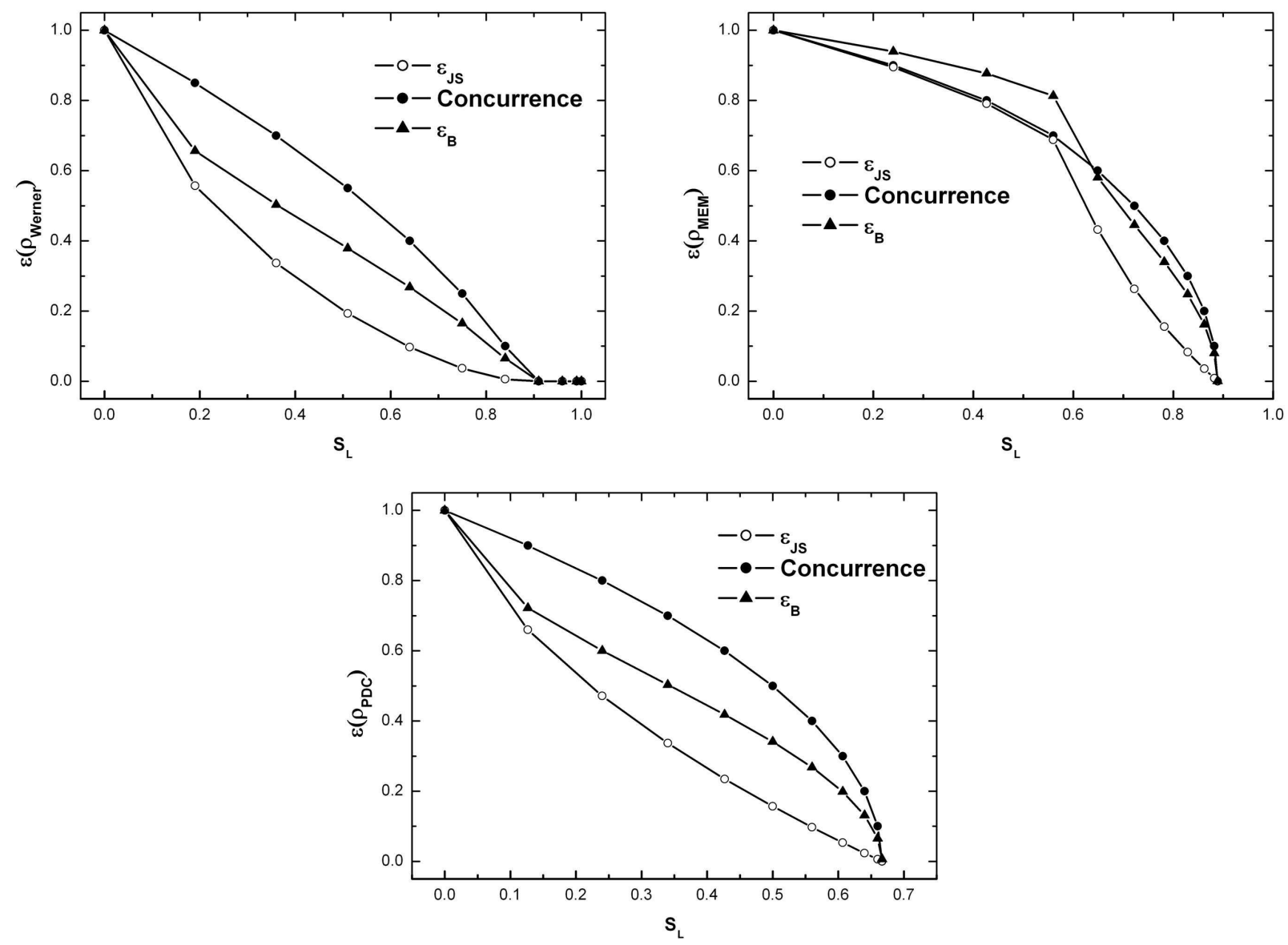

FIG. 2: Comparison between the concurrence, the JS- measure, and the measure $\mathcal{E}_{B}$ induced by the Bures metric. The task is accomplished for (a) Werner, (b) MEM, and (c) PDC states.

measure $\mathcal{E}_{B}$ of Vedral et al., with an important difference: the former is much more easy to compute than the later.

\section{Acknowledgments}

This work was partially supported by the MEC grant FIS2005-02796 (Spain) and FEDER (EU) and by CONICET (Argentine Agency). AB and APM acknowledge support from MEC through FPU grant AP-2004-2962 and contract SB-2006-0165. PWL wants to thank SECyT-UNC (Argentina) for financial support. We thank J. Batlle and Prof. A. R. Plastino for using his Hilbert-Monte Carlo numerical program.

[1] G. Lindblad, Comm. Math. Phys. 33 (1973) 305.

[2] A.P. Majtey et al., Phys. Rev. A 72 (2005) 052310.

[3] C. Rao, IMS-Lectures Notes 10 (1987) 217.

[4] J. Lin, IEEE Trans. Inf. Theory 371 (1991) 145.

[5] A.P. Majtey et al., Eur. Phys. J. D 32 (2005) 413.

[6] V. Vedral and M.B. Plenio, Phys. Rev. A 57 (1998) 1619.

[7] P.W. Lamberti et al., On the metric character of the quantum Jensen-Shannon divergence, arXiv/0801.1586.

[8] M.A. Nielsen and I. L. Chuang, Quantum Computation and Quantum Information (Cambridge University Press, 2000).

[9] H.-K. Lo et al., (Eds.), Introduction to Quantum Computation and Information (World Scientific, River Edge, 1998). 
[10] D. Bouwmeester et al., (Eds.), The Physics of Quantum Information (Springer-Verlag, Berlin, 2000).

[11] I. Bengtsson and K. Życzkowski, Geometry of Quantum States (Cambridge University Press, 2006).

[12] V. Vedral et al., Phys. Rev. Lett. 78 (1997) 2275.

[13] J. Eisert et al., J. Phys. A 36 (2003) 5605.

[14] C. Witte and M. Trucks, Phys. Lett. A 257 (1999) 14.

[15] R.F. Werner, Phys. Rev. A 40 (1989) 4277.

[16] W.J. Munro et al., Phys. Rev. A 64 (2001) 030302. M. Barbieri et al., Phys. Rev. Lett. 92 (2004) 177901.

[17] A. Cabello et al., Phys. Rev. A 72 (2005) 052112. 